\title{
A Comparison of Three Rough Surface Classifiers
}

\author{
G. McGunnigle and M.J. Chantler \\ Department of Computing and Electrical Engineering \\ Heriot Watt University \\ Riccarton \\ Edinburgh, EH14 4AS, UK \\ gmg@cee.hw.ac.uk
}

\begin{abstract}
In this paper texture analysis techniques are used to segment rough surfaces into regions of homogeneous texture. The performance of three rough surface classifiers was assessed and compared. The classifiers differ in their discrimination as well as their input and computational requirements. Experiments were used to identify the failure modes of the classifiers and to identify which classifier is best suited to a particular task. A series of guidelines for the choice of classifier are presented and justified.
\end{abstract}

\section{Introduction}

The visual texture of a surface is caused by the interaction of light with either variations in the surface's reflection characteristics, or with the surface topography. This paper is limited to the second source of texture, and deals with the classification of rough surfaces on the basis of their image texture. Although many texture techniques have been applied implicitly to this form - the majority of Brodatz textures [1] contain at least a component due to surface topography-little work has been carried out on the phenomena associated with this group. One characteristic of rough surface textures is that the appearance of the surface is a function of the illuminant direction as well as of the surface topography [2] [3] [4] [5]. For instance, rotation of a directional surface is not equivalent to rotation of its image,[6], Figure 2. A surface rotation invariant classifier must take this effect into account.

The effect of directional illumination on classification can be reduced by decreasing the (slant) angle between the light source and the vertical-though of course this suppresses the visible texture. An alternative approach is to classify on the properties of the surface rather than those of the image. Photometric stereo allows the estimation of surface derivatives using several images of the same scene captured under different illumination conditions. Critically, it does not need the smoothness constraint required by most singleimage shape from shading algorithms. Figure 3 shows a series of scatter plots of the estimated surface derivatives, $p$ and $q$ corresponding to the image series (Figure 2). The joint distribution of $p$ and $q$ is invariant, apart from a rotation. This makes the surface derivatives an attractive feature for classification. 


\begin{tabular}{|l|c|c|c|}
\hline Classifier & Point & IRIS & SiRIS \\
\hline User Time (s) & 8 & 151 & 158 \\
\hline
\end{tabular}

Table 1: CPU times of feature extraction (includes surface estimation.)

In [2] a classifier (Point) was proposed that extracted rotation invariant features which encapsulated surface roughness and directionality from the point statistics of the surface derivative field. This corresponds to measuring the local distribution of $p$ and $q$ and estimating its major and minor axes. We have concluded that the norm of the surface derivatives, although a less powerful feature, is more robust to errors in surface estimation. In [6] a Surface Rotation InSensitive (SiRIS) classifier was proposed that used isotropic Gabor filters and was insensitive to surface directionality and therefore to surface rotation. The algorithm first decomposes the surface derivative fields into a series of bandlimited fields, before calculating the norm of each field. The third technique investigated is the Image Rotation InSensitive (IRIS) classifier. This is based on the scheme proposed in [7], though in this paper it is used to classify on a pixel by pixel basis rather than to classify regions. SiRIS is almost identical to IRIS except that IRIS operates on image data rather than inferred surface characteristics. The classifiers are summarised in Figure 1.

POINT

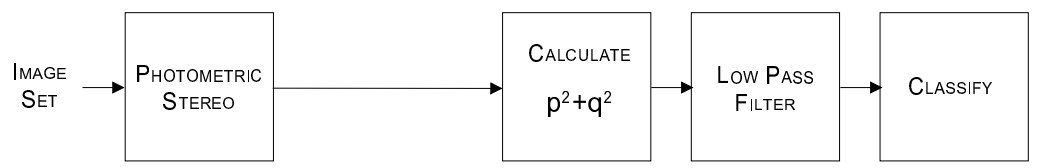

SIRIS

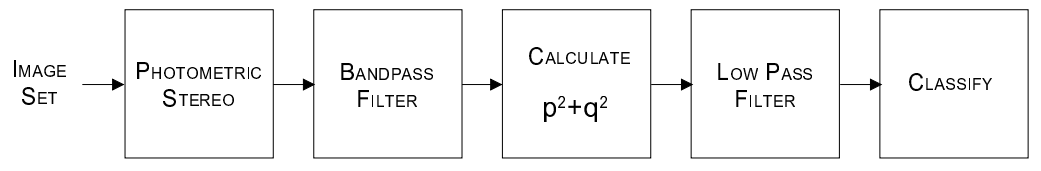

IRIS

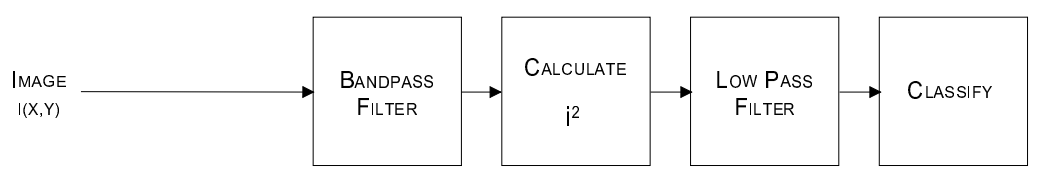

Figure 1: The three test classifiers.

The classifiers differ in their requirements for data and computation. The IRIS scheme requires only one image; the Point and SiRIS classifiers require three, and in this work we have used four. The Point classifier requires least computation, SiRIS requires only marginally more than the IRIS classifier due to the efficient use of the Fast Fourier Transform, (Table 1), see [6]. Given the relative costs of the classifiers it is desirable to identify when the extra expenditure is necessary or useful.

This paper compares the ability of the classifiers to distinguish and segment textured surfaces in an inspection task. We do not try to mimic the human visual system. In many inspection tasks the classifier deals with surfaces that have been formed by similar physical processes - that is they differ in the degree, rather than the type, of processing. The experimental approach of this paper is to test the ability of the classifiers to distinguish 

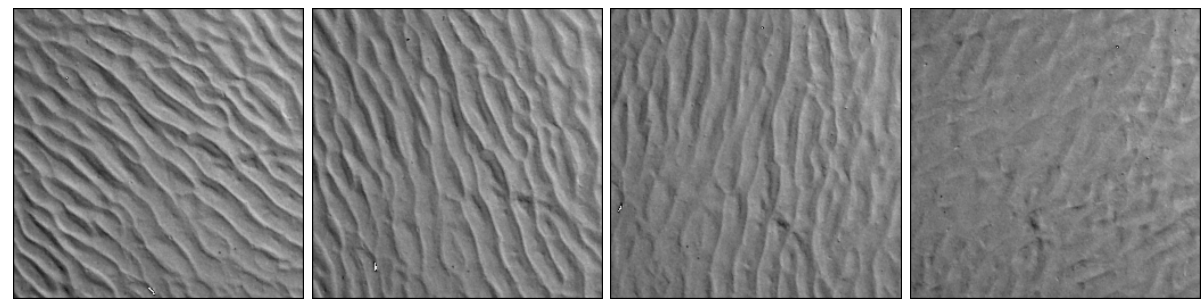

Figure 2: Directional surface rotated clockwise in $30^{\circ}$ increments.

similar surfaces. The textures used in this paper are grouped in montages according to their underlying physical cause. We have tried to use sets of surfaces that are similar, but which show a gradation in appearance. The test surfaces are used to assess the relative performance of the classifiers as well as their robustness to surface rotation.
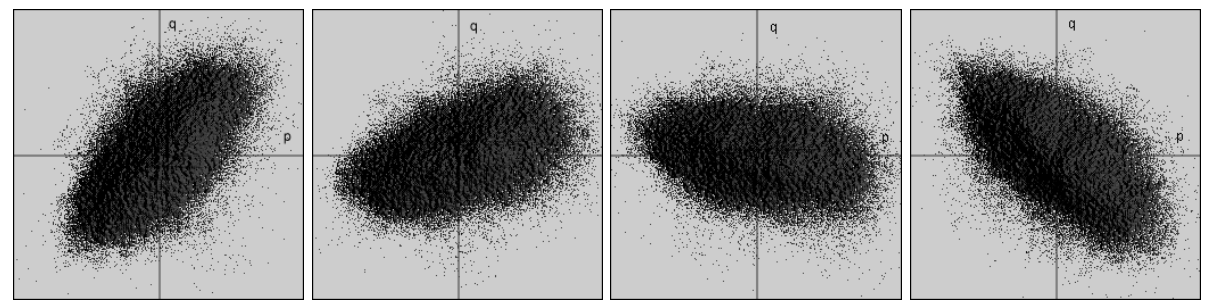

Figure 3: Distribution of facet slope for the rotated directional surface.

In this paper we have found that the Point classifier is the least able to discriminate between surfaces. The SiRIS classifier performs better than the IRIS classifier. Most significantly we have confirmed that the SiRIS and Point classifiers are surface rotation invariant; and that IRIS is not.

Although the Point classifier is the worst classifier tested we do not rule it out for applications: it's simplicity would make it attractive where fast detection, or a fast, coarse segmentation, is required. For isotropic surfaces SIRIS improves on IRIS with little increase in computation - though whether the improvement justifies the more complex imaging process depends on the application. For directional surfaces that are always presented at the same orientation and when the lighting can be optimised, we recommend the IRIS classifier. If the orientation of a directional surface cannot be predicted-we conclude that IRIS is insufficient and a photometric approach such as SiRIS is necessary.

\section{Method}

The classifiers' discrimination and their robustness to surface rotation were assessed on natural surfaces.

The test surfaces are grouped into one of four montages, depending on the physical process that formed them. Fracture surfaces (Figure 4) were formed by impact fractures of solid blocks of plaster. They vary from the relatively smooth, ceramic-like fracture of Fracture 1 to the much rougher, more fractal, Fracture 4 surface. Deposit surfaces (Figure 
5) were formed by the deposition of plaster powder on a plaster flat. These surfaces differ in the amount of powder deposited on the surface. Ground surfaces (Figure 6) were formed by grinding plaster flats. Ground 1 was prepared by grinding in two orthogonal directions; the other surfaces in this group are unidirectional and vary because of the different length of grinding stroke used in their preparation. Ripple surfaces (Figure 7) were formed by wave action and differ in the directionality and frequency.

To allow comparison, all the classifiers use the same training data and the same low pass filter (a Gabor filter with centre frequency of 0 and a standard deviation of 4 cycles per image). The IRIS and SiRIS classifiers also share the same sampling of the frequency spectrum: Gabor filters with centre frequencies of 0, 64, 128, 192 and 256 cycles per image (c/i) and standard deviation of $32 \mathrm{c} / \mathrm{i}$.

All of the classifiers considered ignore phase and discriminate on a sampling of the power spectrum of either the image or the surface derivatives. A stationary random process may be completely characterised by its power spectrum if the process is Gaussian. The assumption that the surface height distributions are Gaussian is common in the rough surface literature. Experimental results suggest that, although not universal, the Gaussian assumption is valid for many natural surfaces [10]. Since differentiation is a linear operation, the assumption also holds for surface derivatives; furthermore, since Lambertian reflection may be modelled as a linear operator for surfaces composed of facets of moderate slope [11], this assumption can be extended to the distribution of image intensities. In this paper, surfaces are assumed to be characterised solely by their power spectrum.

The surfaces were illuminated from a slant of $50^{\circ}$, and tilt angles of $45^{\circ}, 135^{\circ}, 225^{\circ}$ and $315^{\circ}$. The surfaces are made of plaster and are near-Lambertian. This simplifies photometric estimation, however, photometric stereo has been applied to a much wider range of surface reflectance functions both with, and without, prior calibration, [13] [14]. In the experiments each classifier was trained on one sixteenth of the image. In the case of rotation experiments the classifiers were trained prior to rotation. The experiments had two aims: to assess the classifiers' discrimination and to test the robustness of the classifiers to surface rotation.

The surfaces are used in four experiments. The first experiment assesses the ability of the classifiers to segment montages of similar surfaces, Table 2. The remaining experiments test the robustness of the classifiers to surface rotation. The Fracture 1 sample is too small to maintain the necessary image size throughout rotation. We combine Fractures 2 and 4 and Deposits 1 and 4 to form the Iso montage. The classifiers are trained at $0^{\circ}$ of rotation and tested on surfaces rotated in $30^{\circ}$ increments. At each rotation a photometric image set was captured under the same conditions as the earlier experiments.

\section{Results and Discussion}

In experiment 1 the ability of the classifiers to discriminate between closely related surfaces was tested. The percentage of pixels misclassified is shown in Table 2. The segmented Deposit montage is shown in Figures 8-10. For all montages the SiRIS classifier was the most effective and the Point classifier was the worst. However, except for the Ripple montage, the Point classifier is performing a degree of discrimination; and this may be adequate for some applications. Both the simulations and the experimental results indicate that SiRIS is a more robust classifier than IRIS. 

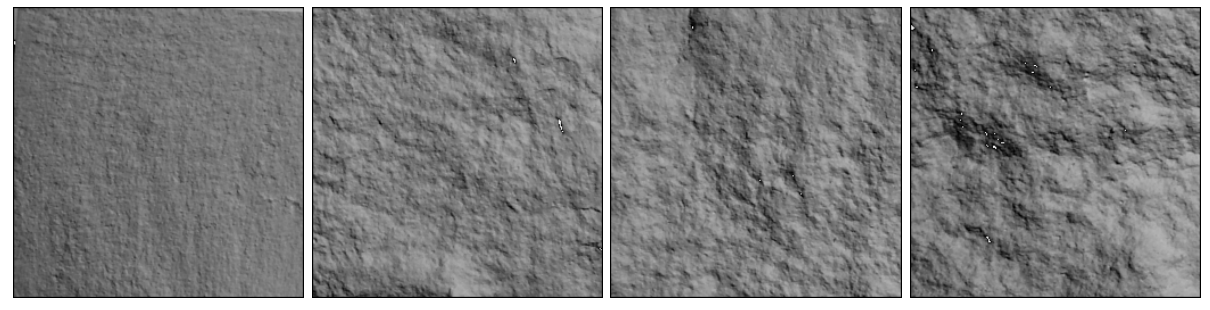

Figure 4: Fracture surfaces.
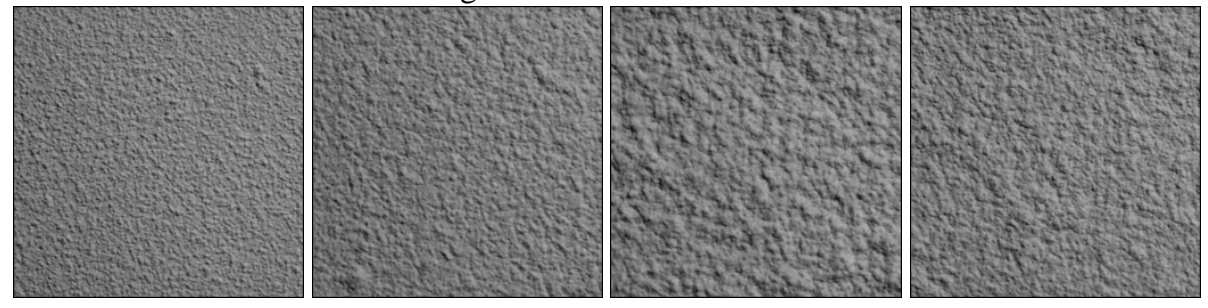

Figure 5: Deposit surfaces.
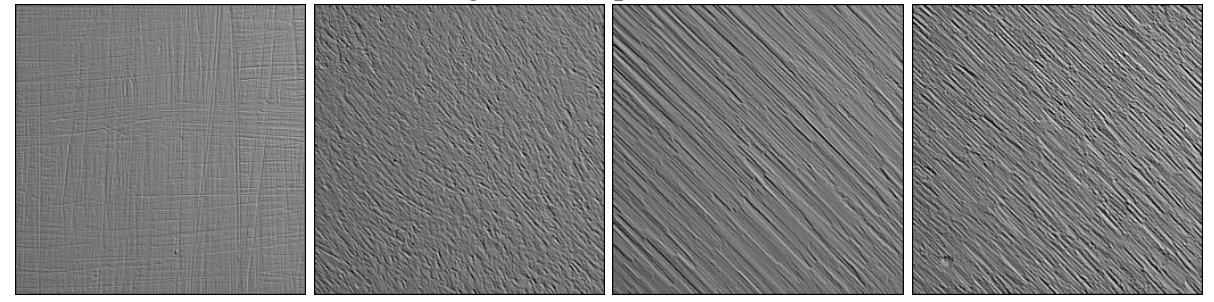

Figure 6: Ground surfaces.
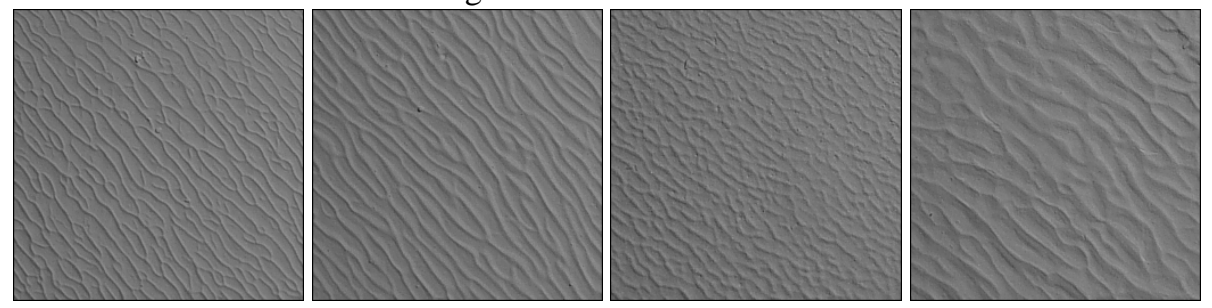

Figure 7: Ripple surfaces. 


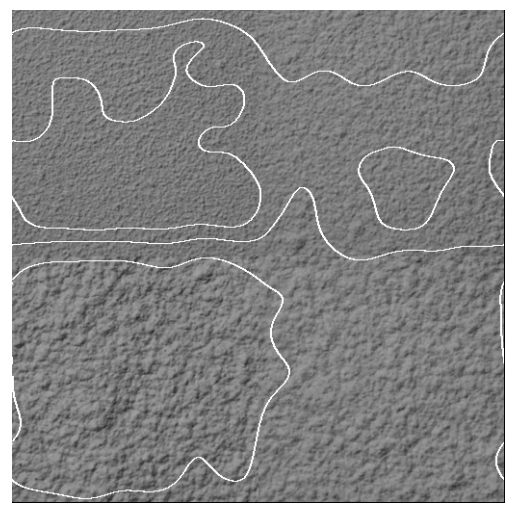

Figure 8: Segmentation of Deposit montage by Point classifier.

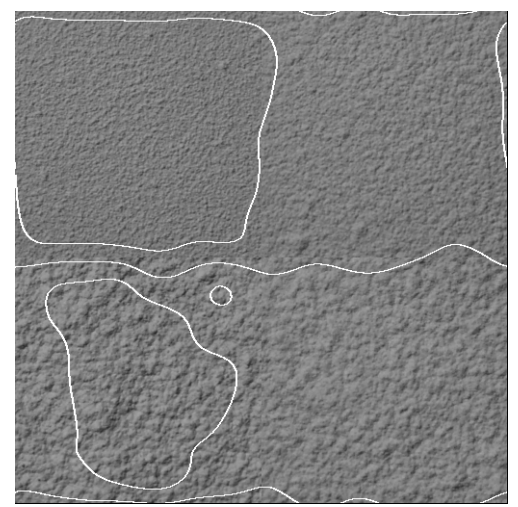

Figure 9: Segmentation of Deposit montage by IRIS classifier.

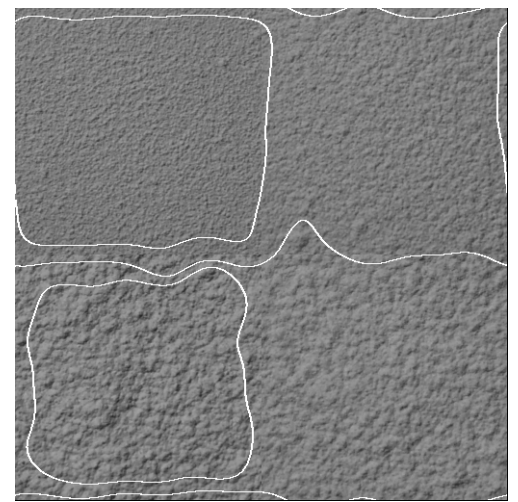

Figure 10: Segmentation of Deposit montage by SiRIS classifier. 


\begin{tabular}{|l|c|c|c|}
\hline Montage & Point & IRIS & SiRIS \\
\hline Fracture & 31.72 & 24.57 & 18.31 \\
Deposit & 30.87 & 18.96 & 12.15 \\
Ground & 40.42 & 21.23 & 14.20 \\
Ripple & 68.83 & 14.44 & 12.95 \\
\hline
\end{tabular}

Table 2: Experiment 1: Classifier error rate (\%).

Experiment 2 tests the classifiers' robustness to the rotation of isotropic surfaces. Unsurprisingly, all the classifiers are unaffected by rotation (Figure 11). The relative accuracies are similar to those found for the isotropic montages in experiment 1 , that is all the classifiers are able to discrimate to some degree.

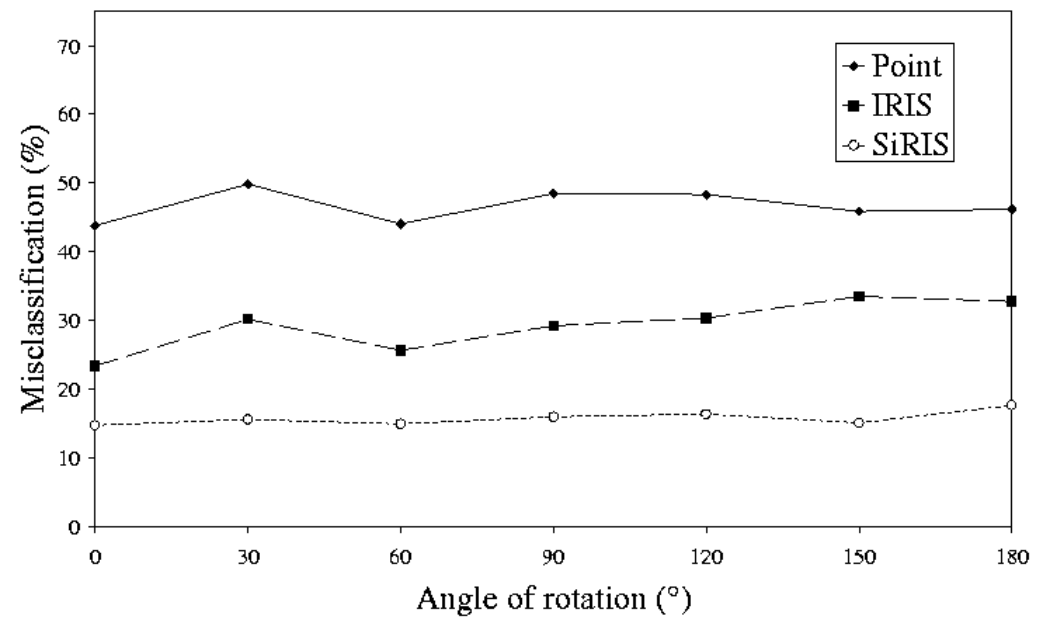

Figure 11: Experiment 2: Classification accuracy with rotated montage of isotropic (fracture and deposit) surfaces.

Experiments 3 and 4 (Figures 12 and 13) test the classifiers' robustness to rotation of directional surfaces. In both experiments the IRIS classifier fails catastrophically as the surface is rotated. As in experiment 1 the Point classifier is completely unable to discriminate any of the Ripple textures. It is able to discriminate between the Ground surfaces - though it does show a degree of sensitivity to rotation: classification is most accurate when the surface directionality is aligned with a pair of illuminants. The SiRIS classifier gives the lowest misclassification and maintains a low level in both experiments. 


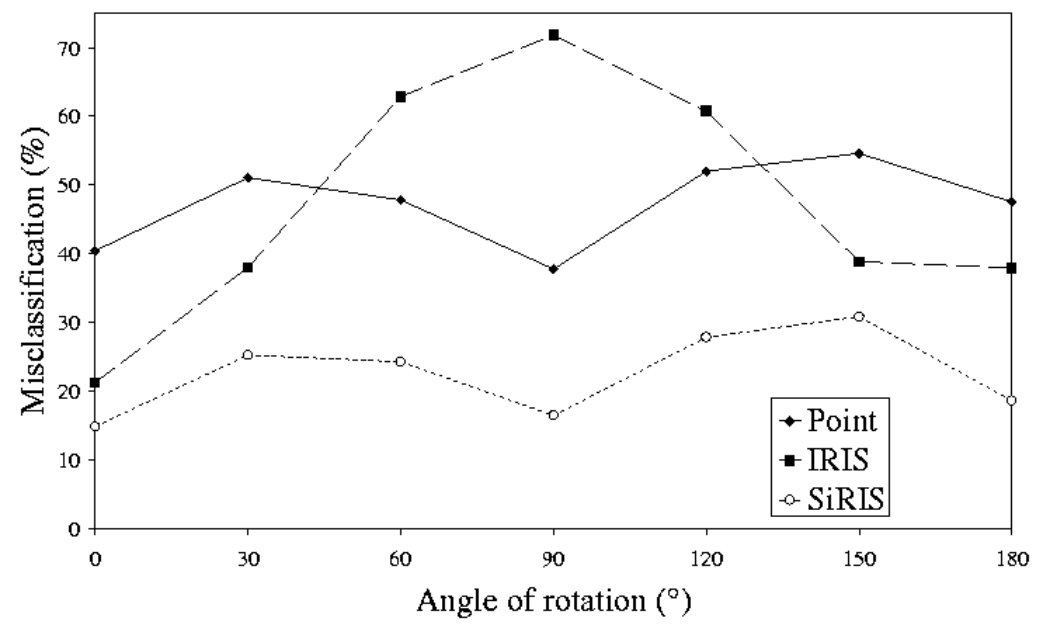

Figure 12: Experiment 3: Classification accuracy with Rotated Ground montage.

\section{Conclusions}

In this paper three techniques that classify rough surfaces by their appearance were compared. Experiments on natural surfaces indicate that the SiRIS classifier is the most effective. In most cases the IRIS classifier is slightly poorer; however, if the test surfaces are directional, and their orientation is unpredictable, the IRIS classifier can fail catastrophically. The Point technique has the least ability to distinguish surfaces. It is able, however, to discriminate to some degree all the surfaces except those in the Ripple montage.

The choice of classifier is specific to the application. Where a rapid, rough classification is required, the computational simplicity of the Point classifier makes it an attractive option. If greater accuracy is required then one of the spectral classifiers should be used. If the classifier deals with directional surfaces that are presented at arbitrary orientations then the IRIS classifier is inadequate and SiRIS should be used. If the surfaces are isotropic or are directional but of known orientation, the choice is less clear. In these cases SiRIS does give more accurate classification than IRIS with no significant increase in computation, however, it does require a photometric image set. Whether the improvement in accuracy outweighs the extra imaging requirements depends on the application

\section{References}

[1] P. Brodatz Textures: a phtographic album for artists and designers, Dover, New York, 1966. 


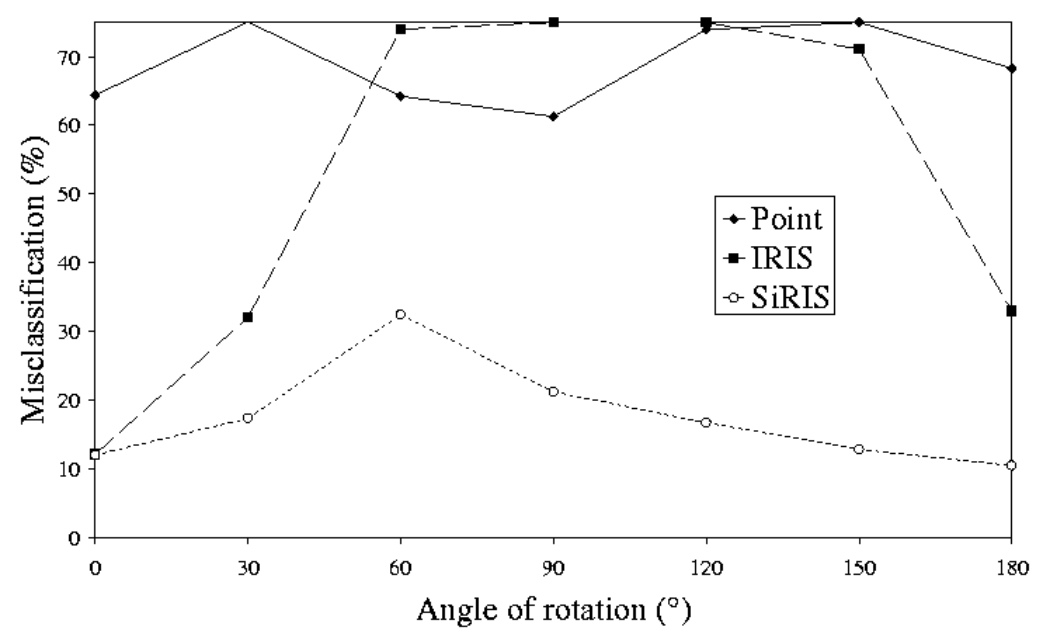

Figure 13: Experiment 4: Classification accuracy with rotated Ripple montage.

[2] G. McGunnigle, M.J. Chantler, Rough surface classification using point statistics from photometric stereo, Pattern Recognition Letters 21 (2000) 593-604.

[3] K.J. Dana, B. van Ginneken, S.K. Nayar, J.J. Koenderink Reflectance and texture of real world surfaces. ACM Transactions on Graphics, Vol. 18, No.1, pp.1-34, January 1999

[4] P.-h. Suen, G. Healey The Analysis and Recognition of Real-World Textures in Three Dimensions. IEEE Transactions on Pattern Analysis and Machine Intelligence, May 2000, Vol.22 No.5 pp.491-503

[5] K.J. Dana, S.K. Nayar Correlation Model for 3D Textures, ICCV '99, pp. 1061-1067, September 1999.

[6] G. McGunnigle, M.J. Chantler, Rotation invariant classification of rough surfaces. IEE Proceedings Vision, Image and Signal Processing, Vol. 146, Number 6, pp. 345352, December 1999.

[7] R. Porter, N. Canagarajah, Robust rotation invariant texture classification: wavelet, Gabor and GMRF based schemes IEE Proceedings Vision, Image and Signal Processing, Vol. 144, Number 3, pp. 180-188, 1997

[8] R.S. Sayles, T.R. Thomas, Surface topography as a nonstationary random process, Nature, Vol.271, 2 February, 1978, pp.431-434.

[9] D.J. Mulvanney, D.E. Newland, K.F. Gill, A Complete Description of Surface Texture Profiles, Wear 132 (1989) pp.173-182. 
[10] J.A. Ogilvy, Theory of Wave Scattering from Random Rough Surfaces, Publisher Adam Hilger, 1991.

[11] P. Kube, A.P. Pentland, On the Imaging of Fractal Surfaces, IEEE Trans. Pattern Analysis and Machine Intelligence, 10(5) pp.704-707, 1988.

[12] R. Woodham, Photometric method for determining surface orientation from multiple images. Optical Engineering, Jan./Feb. 1980, Vol.19 No.1, pp.139-144.

[13] K.V. Rajaram, G. Parthasarth, M.A. Faruqui, A Neural Network Approach to Photometric Stereo Inversion of Real-World Reflectance Maps for Extracting 3-D Shapes of Objects, IEEE Trans. on Systems, Man and Cybernetics, Vol.25, No.9, Sept. 1995, pp.1289-1300.

[14] G. Kay, T. Caelli, Estimating the Parameters of an Illumination Model Using Photometric Stereo, Graphical Models and Image Processing, Vol.57, No.5 Sept. 1995, pp.365-388. 